Pacific Journal of Mathematic 


\title{
EFFECTIVE DIVISOR CLASSES AND BLOWINGS-UP OF $P^{2}$
}

\author{
JEFFREY A. ROSOFF
}

Let $X_{n} \stackrel{\pi}{\rightarrow} \boldsymbol{P}^{2}$ be the monoidal transformation of the (complex) projective plane centered at distinct points $P_{1}, \cdots, P_{n}$ of $P^{2}$. We recall that the Néron-Severi group of $X_{n}$ is freely generated by the divisor class $[L]$ of the proper transform $L$ of a line in $\boldsymbol{P}^{2}$ and by the classes $\left[E_{i}\right]$ of the "exceptional" fibers $E_{\text {, over }} P_{i}$; the intersection pairing is given by

$$
[L]^{2}=1 ;[L] \cdot\left[E_{i}\right]=0 ;\left[E_{\imath}\right] \cdot\left[E_{j}\right]=-\delta_{\imath, j} .
$$

Let $\mathscr{L}\left(X_{n}\right)$ denote the monoid of elements $F$ in the NéronSeveri group with the property that $F$ contains an effective divisor. In this paper we

(1) construct a finite generating set for $\mathscr{H}\left(X_{n}\right)$ for $n \leqq 8$, and give a particularly simple geometric description of the generators when $P_{1} \cdots P_{n}$ are in "general position";

(2) show that, for $n \geqq 9, \mathscr{C}\left(X_{n}\right)$ need not be finitely generated, despite the finite generation of the whole Néron-Severi group;

(3) prove the related result that if a nonsingular surface $X$ contains an infinite number of exceptional curves of the first kind, then $X$ is necessarily rational.

We will let $K_{X_{n}}$ denote the cannonical class on $X_{n}$; it is given by $K_{X_{n}}=\pi * K_{r^{2}}+\Sigma\left[E_{i}\right]=-3[L]+\Sigma\left[E_{i}\right]$. We observe that, for $n \leqq 9$, the anti-cannonical class $-K_{X_{n}}$ contains an effective divisor (which will also be denoted by $-K_{X_{n}}$ when no confusion is possible), since $H^{0}\left(X_{n}, \check{\omega}_{X_{n}}\right)$ can be regarded as the (complex) vector space of homogeneous forms in 3 variables of degree 3 vanishing at the points $P_{1} \cdots P_{n}$.

Lemma 1. Let $X$ be any nonsingular rational surface, and let $C$ be a curve on $X$ with $p_{a}(C) \geqq 1$. Then $[C]+K_{X}$ is an effective class.

Proof. The short exact sequence of $\mathcal{O}_{x}$-modules

$$
0 \longrightarrow O_{X}(-C) \longrightarrow O_{X} \longrightarrow O_{C} \longrightarrow 0
$$

yields, using Serre-duality and the rationality of $X, \operatorname{dim} H^{\circ}\left(X, O_{X}(C) \otimes\right.$ $\left.\omega_{X}\right)=\operatorname{dim} H^{2}\left(X, \mathscr{O}_{X}(-C)\right)=\operatorname{dim} H^{1}\left(C, \mathscr{O}_{C}\right)=p_{a}(C)$.

Recall that, for $n \leqq 8$, the points $P_{1} \cdots P_{n}$ of $\boldsymbol{P}^{2}$ are in general 
position if no three $P_{i}$ are collinear and if no six of them lie on a conic.

THeOREM 1. Let $X_{n} \rightarrow \boldsymbol{P}^{2}$ be the monoidal transformation of $\boldsymbol{P}^{2}$ centered at $P_{1} \cdots P_{n}$, with $n \leqq 8$ and $P_{1} \cdots P_{n}$ in general position. Then $\mathscr{L}\left(X_{n}\right)$ is finitely generated, the generators being the classes of divisors on the following list:

(Note: $g(n)=$ number of generators of $\mathscr{C}\left(X_{n}\right)$ ).

\begin{tabular}{|c|c|c|c|}
\hline$n$ & $g(n)$ & Divisor & Description \\
\hline \multirow[t]{2}{*}{1} & 2 & $E_{1}$ & Exceptional curve \\
\hline & & $L-E_{1}$ & Proper transform of a line through $P$ \\
\hline \multirow{2}{*}{$2,3,4$} & $2,6,10$ & $E_{i}(1 \leqq i \leqq n)$ & Exceptional curve \\
\hline & Respt. & $L-E_{i}-E_{j}(1 \leqq i<j \leqq n)$ & $\begin{array}{l}\text { Proper transform of the line through } \\
\quad P_{i} \text { and } P_{j}\end{array}$ \\
\hline \multirow[t]{3}{*}{5} & 16 & $E_{i} \quad(1 \leqq i \leqq 5)$ & Exceptional curve \\
\hline & & $L-E_{i}-E_{j}(1 \leqq i<j \leqq 5)$ & $\begin{array}{l}\text { Proper transform of the line through } \\
P_{i} \text { and } P_{j}\end{array}$ \\
\hline & & $2 L-\sum E_{i}$ & $\begin{array}{l}\text { Proper transform of the conic through } \\
\quad \text { all }\left\{P_{i}\right\}\end{array}$ \\
\hline \multirow[t]{3}{*}{6} & 27 & $E_{i} \quad(1 \leqq i \leqq 6)$ & Exceptional curve \\
\hline & & $L-E_{i}-E_{j}(1 \leqq i<j \leqq 6)$ & $\begin{array}{l}\text { Proper transform of the line through } \\
P_{2} \text { and } P_{j}\end{array}$ \\
\hline & & $2 L-\sum_{i \neq k} E_{\imath}(1 \leqq k \leqq 6)$ & $\begin{array}{l}\text { Proper transform of the conic through } \\
\quad \text { all }\left\{P_{i}\right\} \text { except } P_{k}\end{array}$ \\
\hline \multirow[t]{4}{*}{7} & 56 & $E_{i} \quad(1 \leqq i \leqq 7)$ & Exceptional curve \\
\hline & & $L-E_{i}-E_{j}(1 \leqq i<j \leqq 7)$ & $\begin{array}{l}\text { Proper transform of the line through } \\
P_{i} \text { and } P_{j}\end{array}$ \\
\hline & & 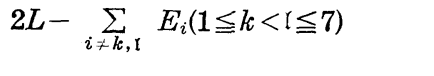 & $\begin{array}{l}\text { Proper transform of the conic through } \\
\text { all points }\left\{P_{i}\right\} \text { except } P_{k} \text { and } P_{i}\end{array}$ \\
\hline & & $3 L-2 E_{j}-\sum_{i \neq j} E_{i}(1 \leqq j \leqq 7)$ & $\begin{array}{l}\text { Proper transform of a cubic through } \\
\text { all } P_{i} \text { and with a double point at } P_{j}\end{array}$ \\
\hline \multirow[t]{8}{*}{8} & 241 & $E_{i} \quad(i=1 \cdots 8)$ & Exceptional curve \\
\hline & & $L-E_{i}-E_{j}(1 \leqq i<j \leqq 8)$ & $\begin{array}{l}\text { Proper transform of the line through } \\
\quad P_{i} \text { and } P_{j}\end{array}$ \\
\hline & & $2 L-\sum_{i \neq j, k \cdot 1} E_{\imath}(1 \leqq j<k<\mathfrak{l} \leqq 8)$ & $\begin{array}{l}\text { Proper transform of the conic through } \\
\text { all }\left\{P_{i}\right\} \text { except } P_{j}, P_{k} \text { and } P_{\mathfrak{l}}\end{array}$ \\
\hline & & $\begin{array}{c}3 L-2 E_{k}- \\
j \neq k)\end{array}$ & $\begin{array}{l}\text { Proper transform of a cubic through } \\
\text { all points }\left\{P_{i}\right\} \text { except } P_{j} \text {, and with } \\
\text { a double point at } P_{k}\end{array}$ \\
\hline & & $\begin{array}{l}4 L-2 E_{j}-2 E_{k}-2 E_{\mathfrak{l}} \\
-\sum_{i \neq j, k, \mathfrak{l}} E_{i}(1 \leqq j<k<\mathfrak{l} \leqq 8)\end{array}$ & $\begin{array}{l}\text { Proper transform of a quartic through } \\
\text { all }\left\{P_{i}\right\} \text { with double points at } P_{j}, P_{k} \\
\text { and } P_{\mathfrak{l}}\end{array}$ \\
\hline & & $5 L-\sum_{i \neq j, k}-E_{k}-2$ & $\begin{array}{l}\text { Proper transform of a quintic through } \\
\text { all }\left\{P_{i}\right\} \text { and with double points at } \\
\text { all but } P_{j} \text { and } \mathrm{P}_{k}\end{array}$ \\
\hline & & $6 L-3 E_{k}-2 \sum_{i \neq k} E_{i}(1 \leqq k \leqq 8)$ & $\begin{array}{l}\text { Proper transform of a sextic with a } \\
\text { triple point at } P_{k} \text { and with double } \\
\text { points at } P_{i}, \forall i \neq k\end{array}$ \\
\hline & & $3 L-\sum_{i=1}^{8} E_{\imath}$ & Anti-cannonical curve \\
\hline
\end{tabular}


REMARK. For $n=6$, we see that the generators of the monoid for the cubic hypersurface in $\boldsymbol{P}^{3}$ are the classes of the classical twenty-seven lines on $X_{6}$. More generally, the classes of the divisors listed above are, for $2 \leqq n \leqq 7$, precisely the classes of all rational curves on $X_{n}$ with self-intersection -1 . [1, Th. 26.2].

Before proving the theorem, we will first prove

Lemma 2. Let $X_{n}$ be as in the theorem. Suppose that $C$ is any curve on $X_{n}$ for $1 \leqq n \leqq 7$, or that $C$ is a curve on $X_{8}$ whose class is not represented above for $n=8$. Then for any divisor $\mathscr{L}$ on the above list, $\operatorname{dim} H^{2}\left(X_{n}, \mathscr{O}_{X_{n}}(C-\mathscr{L})\right)=0$.

Proof. [Case 1: $n \leqq 7]$. A look at the proposed generating set of $\mathscr{C}\left(X_{n}\right)$ shows that, given $\mathscr{L}$ as above, there is an effective nontrivial divisor $D$ such that $-K_{X_{n}}=[\mathscr{L}]+[D]$. Therefore $0=$ $\operatorname{dim} H^{0}\left(X_{n}, \omega_{X_{n}} \otimes \mathscr{O}_{X_{n}}(\mathscr{L})\right)=\operatorname{dim} H^{0}\left(X_{n}, \omega_{X_{n}} \otimes \mathcal{O}(\mathscr{L}-C)\right)$, and the result follows by duality.

[Case 2: $n=8$ ]. Again, we will use duality and show that $\operatorname{dim} H^{0}\left(X_{8}, \omega_{X_{8}} \otimes O_{X_{8}}(\mathscr{L}-C)\right)=0$. Suppose the contrary. Then $K_{X_{8}}+[\mathscr{L}]$ must be an effective class for some $\mathscr{L}$, and we may clearly assume that $[\mathscr{L}] \neq-K_{X_{8}}$. Then either

$$
[\mathscr{L}]=<\left\{\begin{array}{l}
{\left[4 L-2 E_{i}-2 E_{j}-2 E_{K}-\sum_{\mathfrak{l} \neq i, j, k} E_{\mathrm{l}}\right] \text { some } i, j, k, \text { or }} \\
{\left[5 L-E_{i}-E_{j}-2 \sum_{\mathfrak{l} \neq i, j} E_{t}\right] \text { some } i, j, \text { or }} \\
{\left[6 L-3 E_{k}-2 \sum_{i \neq k} E_{i}\right] \text { some } k .}
\end{array}\right.
$$

But by the general position of $P_{1} \cdots P_{8}$, the first two choices for $\mathscr{L}$ do not yield effective classes $[\mathscr{L}]+K_{X_{8}}$; hence $K_{X_{8}}+[\mathscr{L}]$ is of the form $\left[3 L-2 E_{k}-\sum_{i \neq k} E_{i}\right]$.

Now, since $C$ is unequal to any $E_{i}, C \cdot E_{i} \geqq 0$ and we may write $[C]=m[L]-\sum_{i=1}^{8} b_{i}\left[E_{i}\right]$, with $m \geqq 1$ and $b_{i} \geqq 0$. If $K_{X_{8}}+[\mathscr{L}-C]$ is to be effective, we must have $m=1,2$ or 3 . If $m=1$, the general position of the $\left\{P_{i}\right\}$ forces all but two of the $b_{i}$ to be 0 and the nonzero $b_{i}$ to be 1 , making $\left[K_{X_{8}}+\mathscr{L}-C\right]=\left[2 L-\sum c_{i} E_{i}\right]$ with $\sum c_{i} \geqq 6$. This class is not effective since no six of the $\left\{P_{i}\right\}$ lie on a conic. An analogous proof works for $m=2$. If $m=3$ we have, since $[C] \cdot\left[L-E_{i}-E_{j}\right] \geqq 0$ for all $i, j$, three possibilities:

(a) some $b_{i}=3$, all others 0 , or

(b) all $b_{i}$ are 0 or 1 , or

(c) some $b_{i}=2$, all others are 0 or 1 .

Neither (a) nor (b) can occur, as in these cases $K_{X_{8}}+[\mathscr{L}-C]=$ $\sum c_{i}\left[E_{i}\right]$ with some $c_{2}<0$, violating the effectiveness of $K_{X_{8}}+[\mathscr{L}-$ 
$C]$. Similarly, (c) can be dismissed unless $[C]$ is of the form [ $3 L-$ $2 E_{i}-\sum_{k \neq i, j} E_{k}$ ], some $i, j$, which violates the hypothesis that [C] not be represented on the list of divisors in the theorem.

Proof of Theorem 1. Fix a projective embedding of $X_{n}$ into $\boldsymbol{P}^{N}$, some $N \geqq 3$. Then we may speak of the "degree" of a divisor on $X_{n}$ with respect to this embedding. It suffices to show that, for $C$ an effective divisor on $X_{n},[C-\mathscr{L}]$ is an effective class for some divisor $\mathscr{L}$ listed in the theorem; the result will then follow by induction on "degree". Furthermore, for $n=1, \cdots, 7$ we note that $-K_{X_{n}}$ is a sum of classes of divisors listed, while for $n=8$ the anti-cannonical class is included on the list of proposed generators. Hence, by Lemma 1 , we may assume that $C$ is a curve with $p_{a}(C)=0$. Finally, we may assume that $C$ is an irreducible curve whose class is not represented on the list in the theorem.

By Riemann-Roch, together with Lemma 2 and the rationality of $X_{n}$, we have, for $\mathscr{L}$ any divisor on the above list except $-K_{X_{8}}$, $\operatorname{dim} H^{0}\left(X_{n}, O_{X_{n}}(C-\mathscr{L})\right)-\operatorname{dim} H^{1}\left(X_{n}, O_{X_{n}}(C-\mathscr{L})\right)=1 / 2\left(C^{2}-2 \mathscr{L}\right.$. $\left.C-K_{X_{n}} \cdot C\right)$. Since $p_{a}(C)=0$, the adjunction formula applied to $C$ yields $C^{2}=-K_{X_{n}} \cdot C-2$, so we have, for all divisors $\mathscr{L}$ on the list in the theorem except for $-K_{X_{8}}$,

$$
\begin{gathered}
\operatorname{dim} H^{0}\left(X_{n}, O_{X_{n}}(C-\mathscr{L})\right)-\operatorname{dim} H^{1}\left(X_{n}, \mathscr{C}_{X_{n}}(C-\mathscr{L})\right) \\
=\left(-K_{X_{n}} \cdot C\right)-1-(\mathscr{L} \cdot C) .
\end{gathered}
$$

Thus, it suffices to show that for some divisor $\mathscr{L}$ in the above list except for $-K_{X_{b}}$,

$$
\text { (*) }-K_{X_{n}} \cdot C>\mathscr{L} \cdot C+1 \text {. }
$$

The proof of the validity of $(*)$ is, for $n=1, \cdots, 5$, a simplified version of the cases $n=6,7,8$; hence we include only the later cases.

Let $[C]=m[L]-\sum_{i=1}^{n} b_{i}\left[E_{i}\right]$. Since $[C]$ is not represented on the above list, we intersect $C$ with each element on the list to get

$$
\begin{array}{lll}
n=6: & \text { (1) } \quad m \geqq 1 & \text { (3) } m-b_{i}-b_{j} \geqq 0 \forall i \neq j \\
& \text { (2) } b_{i} \geqq 0 \forall i & \text { (4) } 2 m-\sum_{i \neq k} b_{i} \geqq 0 \forall k .
\end{array}
$$

Since $-K_{X_{6}} \cdot C=3 m-\sum_{i=1}^{6} b_{i}$, our condition $\left(^{*}\right)$ to be fulfilled becomes

$$
\left({ }^{*}\right)<\left[\begin{array}{l}
3 m>\sum_{i=1}^{6} b_{i}+b_{k}+1 \text { for some } k, \text { or } \\
2 m>\sum_{k \neq i, j} b_{k}+1 \text { for some } i, j \text { or } \\
m>b_{k}+1 \text { for some } k .
\end{array}\right.
$$


If $m>1$, and if the third inequality of $\left(^{* *}\right)$ fails, then, by conditions (2) and (3) above we have $m=2$ and $b_{k}=1 \forall k$, violating (4) above. If $m=1$, then by (2) and (3) at most one $b_{i}$ can be nonzero, and the first two inequalities of $\left({ }^{* *}\right)$ hold.

$n=7$ we have
(1) $m \geqq 1$
(4) $2 m-\sum_{i \neq j k} b_{i} \geqq 0 \forall j \neq k$
(2) $b_{i} \geqq 0 \forall i$
(5) $3 m-\sum_{j \neq i} b_{j}-2 b_{i} \geqq 0 \forall i$,
(3) $m-b_{i}-b_{j} \geqq 0 \forall i \neq j$

and condition $\left(^{*}\right)$ becomes

$$
(* *)<\left[\begin{array}{l}
3 m>\sum_{i=1}^{7} b_{i}+b_{k}+1 \text { for some } k, \text { or } \\
2 m>\sum_{i \neq j, k} b_{i}+1 \text { for some } j, k, \text { or } \\
m>b_{j}+b_{k}+1 \text { for some } j, k, \text { or } \\
b_{i}>1 \text { for some } i .
\end{array}\right.
$$

Assume that the fourth inequality of $\left({ }^{* *}\right)$ fails. If all $b_{i}$ are 1 , and if the third inequality of $\left(^{* *}\right)$ fails, then $m \leqq 3$. By condition (4) we have $m \geqq 3$, so $m=3$ and $[C]=-K_{X_{7}}$, which we have already seen is a sum of proposed generators of $\mathscr{C}\left(X_{7}\right)$. If some $b_{i}$ is 0 , then conditions (1) $\cdots(4)$ and the first three conditions of $\left({ }^{* *}\right)$ become the same as in the case $n=6$.

$n=8$ writing condition $\left(^{*}\right)$ in terms of $m$ and the $b_{i}(i=1, \cdots, 8)$ and assuming that $\left(^{*}\right)$ does not hold, we have:

( $\alpha)\left|3 m-b_{k}-\sum_{i=1}^{8} b_{i}\right| \leqq 1$ for all $k$

(ß) $\left|2 m-\sum_{i \neq j, k} b_{i}\right| \leqq 1$ for all $j, k$

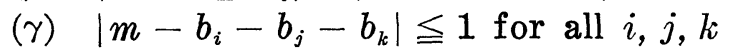

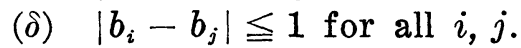

Let $b=\min \left\{b_{i}\right\}$, and $B=\max \left\{b_{i}\right\}$. Note that by $(\delta), 0 \leqq B-b \leqq 1$. Let $r$ of the $b_{i}$ 's have value $b$, and $8-r$ of the $b_{i}$ 's have value $B$. We will obtain our contradiction on a case-by-case basis:

$r=0$. Then by $(\alpha) m-3 B=0$ and $[C]=B\left(-K_{X_{8}}\right), B \in Z$; since $p_{a}(C)=0$ the adjunction formula yields $B^{2}-B+2=0$.

$r=8$. Again by $(\alpha),[C]=b\left(-K_{X_{8}}\right)$.

$r=1$. By $(\beta), m-3 B=0$, and by $(\alpha)|3 m-7 B-2 b| \leqq 1$, contradicting $B-b=1$.

$r=7$. Then $m-3 b=0$ by $\beta$, which is again impossible by $(\alpha)$ and the fact that $B-b=1$ for $r \neq 0,8$.

$r=2$. Since $B-b=1,(\beta)$ implies that $2 m-5 B-b=0$, and

( $\gamma$ ) implies that $m-2 B-b=0$. Thus $B-b=0$, a contradiction.

$r=6$. Again, $(\gamma)$ and $(\beta)$ imply that $B-b=0$. 
$r=3,4,5 . \quad$ By $(\gamma),|m-3 b| \leqq 1$ and $|m-3 B| \leqq 1$, so $B-b=$ 0 , a contradiction.

We now examine the case in which the points $P_{1}, \cdots, P_{n}$, with $n \leqq 8$, of $P^{2}$ are not in general position; in this case the classes of the divisors listed in Theorem 1 may contain reducible curves. For each $n \leqq 8$, let $F_{1} \cdots F_{m}$ be the classes of the formal sums of $L$ and the $\left\{E_{i}\right\}$ listed in Theorem 1 , and let $D_{i} \in F_{i}$ be an effective divisor with the property that the number of distinct components of $D_{i}$ is maximal for effective divisors in $F_{i}$. (Such a divisor $D_{i}$ exists since, for any effective divisor $D \in F_{i}$, \# components of $D \leqq$ $\operatorname{deg} D=\operatorname{deg} E$ for any $E \in F_{i}$.) Write $D_{i}=\sum_{j} n_{i, j} E_{i, j}$ with $n_{i, j}>0$.

Lemma 3. Let $P_{1}, \cdots, P_{8}$ be distinct points of $\boldsymbol{P}^{2}$ in arbitrary position, and let $X_{8} \rightarrow \boldsymbol{P}^{2}$ be the monoidal transformation centered at the $\left\{P_{i}\right\}$. Let $D_{i} \in F_{i}$ be as above, for $n=8$. Then there are only a finite number of divisor classes $F$ on $X_{8}$ with the property that $F$ contains curve $C$ with $p_{a}(C)=0$ and with the property that $\operatorname{dim} H^{2}\left(X_{8}, C_{X_{8}}\left(C-D_{i}\right)\right) \geqq 1$ for some $i$.

Proof. If $\operatorname{dim} H^{2}\left(X_{8}, C_{X_{8}}\left(C-D_{i}\right)\right) \geqq 1$, then, by duality, $K_{X_{8}}+$ $\left[D_{i}\right]-[C]$ must contain an effective divisor, and so must $K_{X_{8}}+F_{i}$. Thus, as in the proof of Theorem $1, K_{X_{8}}+F_{i}$ must be of the form

$$
\begin{aligned}
& {[L]-\left[E_{i}\right]-\left[E_{j}\right]-\left[E_{k}\right], \text { some } i, j, k, \text { or }} \\
& 2[L]-\sum_{\mathfrak{i} \neq i, j}\left[E_{\mathfrak{t}}\right], \text { some } i, j, \text { or } \\
& 3[L]-2\left[E_{k}\right]-\sum_{i \neq k}\left[E_{i}\right], \text { some } k .
\end{aligned}
$$

Hence, if $[C]=m[L]-\sum b_{i}\left[E_{i}\right]$, we must have $0 \leqq m \leqq 3$, and since $p_{a}(C)=0$, the adjunction formula yields $\left(m^{2}-3 m\right)-\sum_{i=1}^{8}\left(b_{i}^{2}-\right.$ $\left.b_{i}\right)=-2$. Clearly with $0 \leqq m \leqq 3$ there are only a finite number of solutions to this diaphantine equation.

Let $R_{1} \cdots R_{k}$ be the divisor classes on $X_{8}$ referred to in Lemma 3 , and let $S_{i} \in R_{i}$ be an effective divisor with maximal number of distinct components. Write $S_{i}=\sum_{j} m_{i, j} Q_{i, j}$, with $m_{i, j}>0$.

THEOREM 2. Let $X_{n} \rightarrow \boldsymbol{P}^{2}$ be the monoidal transformation centered at points $P_{1} \cdots P_{n}$ of $\boldsymbol{P}^{2}$, with $n \leqq 8$ and with the points $\left\{P_{i}\right\}$ in arbitrary positions. Then $\mathscr{C}\left(X_{n}\right)$ is finitely generated, the generators being $\left\{E_{i, j}\right\}$ for $n \leqq 7$, and $\left\{\left[E_{i, j}\right]\right\} \cup\left\{\left[Q_{i, j}\right]\right\}$ if $n=8$.

Proof. [Case 1: $n \leqq 7]$. We will show that, for $C$ an irreducible 
curve on $X_{n}, C-E_{i, j}$ is equivalent to an effective divisor, for some $i, j$. As in the proof of Theorem 1 , we may assume that $p_{a}(C)=0$. Moreover, the proof of Lemma 2 for $n \leqq 7$ did not rely on the general position of the $\left\{P_{i}\right\}$; hence for any curve $C$ on $X_{n}, n \leqq 7$, $\operatorname{dim} H^{2}\left(X_{n}, \mathcal{O}_{X_{n}}\left(C-D_{i}\right)\right)=0$ for all $i$. Thus it suffices to show that

(a) if $p_{a}(C)=0, C$ irreducible and $[C] \neq\left[E_{i, j}\right]$ for all $i, j$, then $\chi\left(\mathcal{O}_{X_{n}}\left(C-D_{i}\right)\right) \geqq 1$ for some $i$, and

(b) $\left[E_{i, j}\right]$ cannot be written nontrivially as a sum of effective divisor classes.

Part (b) follows from the maximality of the number of components of $D_{i}$ for effective divisors in $F_{i}$. For part (a) we note that, since the intersection-theoretic properties of the $\left\{F_{i}\right\}$ are the same as in Theorem 1 , it suffices to show that

$$
\text { (*) }-K_{X_{n}} \cdot C>\left(D_{i} \cdot C\right)+1 \text { for some } i,
$$

with $[C] \neq\left[E_{i, j}\right] \forall i, j$. Writing $[C]=m[L]-\sum_{i=1}^{n} b_{i}\left[E_{i}\right]$ and writing $\left(^{*}\right)$ in terms of $m$ and the $\left\{b_{i}\right\}$, the condition $\left(^{*}\right)$ becomes precisely the condition $\left({ }^{* *}\right)$ of Theorem 1 .

Since $[C] \neq\left[E_{i, j}\right]$ for all $i, j$, we have $C \cdot D_{i} \geqq 0 \forall i$, i.e., the constraints on $m$ and the $\left\{b_{i}\right\}$ are the same as in the proof of Theorem 1. Since the truth of $\left(^{* *}\right)$ depended only on these constraints, we are done.

[Case 2: $n=8$ ]. As in the case $n \leqq 7$, it suffices to show that for $C$ an irreducible curve on $X_{8}$ with $p_{a}(C)=0$, either $C-E_{i j}$ or $C-Q_{i, j}$ is equivalent to an effective divisor. Clearly, if $C \in R_{i}$, for some $i$, then $C-Q_{i, j}$ is equivalent to an effective divisor for some $i, j$. If $C \notin R_{i}$ for any $i$, it suffices to show that, with $C \neq$ $E_{i, j}$ for all $i, j$,

$$
\text { (*) } \chi\left(\mathcal{O}_{X_{8}}\left(C-D_{i}\right)\right) \geqq 1 \text { for some } i \text {. }
$$

Since $C \cdot D_{i} \geqq 0$ for all $i$, the verification of $\left(^{*}\right)$ reduces to the case $n=8$ of Theorem 1 .

In contrast with the above, if $n \geqq 9, \mathscr{M}\left(X_{n}\right)$ need not be finitely generated.

ExAmple. Let $C_{1}$ be a cuspidal cubic curve in $\boldsymbol{P}^{2}$, and let $C_{2}$ be any cubic curve intersecting $C_{1}$ in nine distinct points, none of which is a singular point of $C_{1}$. Let $Y$ be the surface obtained by blowing up $P^{2}$ at $C_{1} \cap C_{2}$. Claim: $\mathscr{C}(Y)$ is not finitely generated.

Let $F_{i}\left(X_{0}, X_{1}, X_{2}\right)$ be the (cubic) defining polynomials of $C_{i}(i=$ 1,2). Then the rational function $F_{1} / F_{2}$ on $\boldsymbol{P}^{2}$ has its only inde- 
terminate points on $C_{1} \cap C_{2}$. Since $C_{1}$ and $C_{2}$ are transversal, the rational function $F_{1} / F_{2}$ pulls back to $Y$ to give a holomorphic map $\phi: Y \rightarrow \boldsymbol{P}^{1}$, with fibers the proper transforms under the blowing up $\pi: Y \rightarrow P^{2}$ of the curves in the pencil generated by $C_{1}$ and $C_{2}$.

Let $Y^{*}$ denote the set $Y-\bigcap_{t \in P^{1}}$ sing $\phi^{-1}(t)$, and let $\phi^{-1}\left(t_{0}\right)$ be the proper transform of the cuspidal curve $C_{1}$. The fibers of an elliptic fibering have been classified by [2, Th. 6.2 and 9.1], along with the possible group structures of the set of nonsingular points; we see by the classification that $\phi^{-1}\left(t_{0}\right) \cap Y^{*}$ has the structure of a torsion-free abelian group, with any point serving as the identity element.

Let $\Gamma$ denote the set of sections of $\phi$ (which necessarily map into $Y^{*}$ ); then after choosing some element of $\Gamma$ (such as one of the nine exceptional curves lying over a point of $C_{1} \cap C_{1}$ ) as an identity element, $\Gamma$ has the structure of an abelian group under pointwise addition (the addition being the group operations on the nonsingular sets of the fibers of $\phi$ ). We have, for each $t \in \boldsymbol{P}^{1}$, a natural evaluation homomorphism

$$
\dot{\psi}_{t}: \Gamma \longrightarrow \phi^{-1}(t) \cap Y^{*} \text {, defined by } \sigma \longrightarrow \sigma(t) \text {. }
$$

Since $\Gamma$ contains at least nine disjoint sections (i.e., the nine exceptional curves lying over $C_{1} \cap C_{2}$ ), the map $\psi_{t_{0}}$ maps $\Gamma$ nontrivially into a torsion-free group, so $\Gamma$ must be infinite.

By [2, Th. 9.2], each $\eta \in \Gamma$ induces a fiber-preserving automorphism

$$
L_{\eta}: Y^{*} \longrightarrow Y^{*} \text {, defined by } L_{\eta}(z)=z+\eta \circ \phi(z) \text {, which }
$$

actually extends to an automorphism of $Y$. Thus, any two elements of $\Gamma$ differ by an automorphism of $Y$.

Hence, the orbits of the exceptional curves lying over $C_{1} \cap C_{2}$ under the action of Aut $(Y)$ yield an infinite number of exceptional curves of the first kind on $Y$. The following fact shows that $\mathscr{M}(Y)$ is not finitely generated, while of course N.S. $(Y) \approx$ $P I C(Y) \approx Z \oplus^{10}$.

Fact. Let $Y$ be any surface containing an infinite number of curves of negative self-intersection. Then $\mathscr{C}(Y)$ is not finitely generated.

Proof. Suppose to the contrary that $\mathscr{L}_{1}, \cdots, \mathscr{L}_{n}$ is a (finite) generating set of $\mathscr{M}(Y)$. To obtain a contradiction it suffices to show that if $C_{i}$ is a fixed curve in the algebraic equivalence class $\mathscr{L}_{i}$, and if $E$ is a curve on $Y$ with negative self-intersection, then 
$E$ must be a component of $C_{i}$, for some $i$. For the curves $C_{i}$ and $E$ as stated, write

$$
[E]=\sum_{i=1}^{n} m_{i=} \mathscr{L}_{i}=\sum_{i=1}^{n} m_{i}\left[C_{i}\right], \text { with } m_{i} \geqq 0 .
$$

Therefore $E^{2}=\sum_{i=1}^{n} m_{i}\left(C_{i} \cdot E\right)$. If $E$ is not a component of $C_{i}$ for any $i$, then the right-hand side of the above equation is nonnegative, which is a contradiction.

REMARK. The elliptic surface constructed above is only one of a large number of known examples of surfaces which contain an infinite number of rational curves with self-intersection -1 and which are obtained by blowing up the projective plane at nine points. For other examples, see [5, p. 164], or [1, p. 407].

REMARK. It is not hard to show, using the projection formula [1, p. 426 A. 4] that if $X \rightarrow Y$ is a monoidal transformation of surfaces, and if $\mathscr{C}(X)$ is finitely generated, then $\mathscr{C}(Y)$ is also finitely generated. Hence $\mathscr{C}\left(X_{n}\right)$ need not be finitely generated for $n \geqq 9$.

In view of the fact used above, the question naturally arises as to which surfaces can contain an infinite number of curves with negative self-intersection. A partial answer is given by a conjecture of A. Kas, a proof of which is provided below:

THEOREM 3. Let $X$ be nonsingular algebraic surface over $C$ which contains an infinite number of exceptional curves of the first kind. Then $X$ is rational.

Proof. Let $\dot{\phi}_{1}, \cdots, \phi_{n}$ be a basis of holomorphic 1-forms on $X$, for $n \geqq 0$. We will first reduce to the case $n=0$.

Case $1 . \quad n \geqq 2$ and $\phi_{i} \wedge \phi_{j} \neq 0$, some $i, j$.

We write the cannonical map $\pi: X \rightarrow \operatorname{Alb}(X)$, given by

$$
z \longrightarrow\left[\int_{P}^{z} \phi_{1}, \cdots, \int_{P}^{z} \phi_{n}\right]
$$

modulo the lattice in $C^{n}$ generated by the $2 n$ vectors

$$
\left[\begin{array}{ll}
\int \dot{\rho}_{1}, & \cdots, \int \dot{\phi}_{n} \\
\Gamma_{i} & \Gamma_{i}
\end{array}\right], \quad i=1, \cdots, 2 n,
$$


where $P$ is a fixed point of $X$ and $\Gamma_{1}, \cdots, \Gamma_{2 n}$ are 1-cycles whose homology classes generate the free subgroup of $H_{1}(X, Z)$.

The hypothesese imply that the Jacobian of the Albanese map $\pi$ has rank 2; hence $\pi$ is generically finite-to-one in the sense that there are only a finite number of points $p \in \operatorname{Alb}(X)$ such that $\operatorname{dim} \pi^{-1}(p)=1$. Let $\left\{p_{1}, \cdots, p_{k}\right\}$ be this finite set, and let $\pi^{-1}\left(p_{i}\right)$ be the divisor $\sum n_{i j} D_{j}$, with $n_{i j}>0$ and $D_{i j}$ irreducible. If $C$ is a rational curve on $X$, then $\pi(C)$ is a single point; hence the number of rational curves on $X$ is bounded by $\sum n_{i j}$. (Actually it is not hard to see that a rational curve on $X$ must be a component of a fixed divisor in the cannonical class of $X$.)

Case 2. $n=1$, or $n \geqq 2$ and $\phi_{i} \wedge \phi_{j}=0 \forall i, j$.

If $n=1$, then $\operatorname{dim} \pi(X)=\operatorname{dim} \operatorname{Alb}(X)=1$. If $n \geqq 2$, the fact that $\phi_{i} \wedge \phi_{j}=0 \forall i, j$ implies that the Jacobian matrix of $\pi$ has rank 1 , and $\operatorname{dim} \pi(X)=1$ in this case as well.

Let $\Delta$ be the curve $\pi(X) \subset \operatorname{Alb}(X)$, and let $\left\{a_{1} \cdots a_{r}\right\} \subset \Delta$ be the (finite) set of points such that $\forall t \in \Delta, \pi^{-1}(t)$ is singular if and only if $t=a_{i}$, some $i$. Let $C$ be a rational curve on $X$ with nonzero self-intersection. Then $\pi(C)$ is a point of $A$, so $C$ is a component of $\pi^{-1}\left(t_{0}\right)$, some $t_{0} \in \Delta$. Since $\left(\pi^{-1}(t)\right)^{2}=0 \forall t$, and since $C^{2} \neq 0, t_{0} \in$ $\left\{a_{1} \cdots a_{r}\right\}$. Thus the number of rational curves on $X$ with nonzero square is bounded by $\sum_{i, j} n_{i, j}$, where $\pi^{*}\left(a_{i}\right)$ is the effective divisor $\sum_{j} n_{i, j} D_{j}$. Therefore, we have reduced to

Case 3. $X$ has no (global) holomorphic 1-forms. For $C$ an exceptional curve of the first kind on $X$, the adjunction formula yields $C \cdot K_{x}=-1$, and so $C \cdot m K_{x}<0 \forall m>0$.

Case 3a. $2 K_{x}$ contains an effective divisor $D$. Then since $D \cdot C<0, C$ must be a component of $D$, and the number of exceptional curves of the first kind on $X$ is bounded by $\sum n_{i}$, where $D=\sum n_{i} D_{i}$, with $D_{i}$ integral and $n_{i}>0$.

Case 3b. $2 K_{x}$ does not contain an effective divisor, i.e., $P_{2}(X)=$ 0 . Since $X$ has no global holomorphic 1-forms, $q(X)=\operatorname{dim} H^{1}(X$, $\left.\mathscr{O}_{x}\right)=0$. Since $q(X)=P_{2}(X)=0, X$ is rational by the classification theorem of Castelnuovo [3. Th. 49]).

REMARK. Among the standard surface types, it is also known that certain $K 3$ surfaces contain an infinite number of -2 curves. In addition, it seems to be a part of the folklore that, for each positive integer $n$, there is an elliptic surface containing an infinite 
number of curves with self-intersection $-n$.

We end this paper with a conjecture, a discussion of which is to appear in the near future:

Conjecture. Let $X$ be a nonsingular algebraic surface of general type. Then $\mathscr{H}(X)$ is finitely generated.

\section{REFERENCES}

1. R. Hartshorne, Algebraic Geometry, Graduate Texts in Math., Springer-Verlag, New York Inc., 1977.

2. K. Kodaira, On compact analytic surfaces II, Annals of Math., 77, No. 3 (1963). 3. - On the structure of complex analytic surfaces IV, Amer. J.Math., 90 (1968), 1048-1066.

4. Yu I. Manin, Cubic Forms: Algebra, Geometry, Arithmetic; North Holland Pub. Co., Amsterdam, 1974.

5. I. R. Saferevic, Algebraic surfaces, Proc. Steklov Inst. Math., 75 (1965), Transl. by Amer. Math. Soc., (1967).

Received September 17, 1979 and in revised form December 16, 1979.

Illinois State University

Normal, IL 61761. 



\section{PACIFIC JOURNAL OF MATHEMATICS}

\section{EDITORS}

DONALD BABBITT (Managing Editor)

University of California

Los Angeles, CA 90024

HUGo RossI

University of Utah

Salt Lake City, UT 84112

C. C. MOORE and ANDREW OGG

University of California

Berkeley, CA 94720
J. DugundJI

Department of Mathematics

University of Southern California

Los Angeles, CA 90007

R. FinN and J. Milgram

Stanford University

Stanford, CA 94305

ASSOCIATE EDITORS
E. F. BECKENBACH
B. H. NeUmanN
F. WOLF
K. YoSHIDA

\section{SUPPORTING INSTITUTIONS}

UNIVERSITY OF BRITISH COLUMBIA

CALIFORNIA INSTITUTE OF TECHNOLOGY

UNIVERSITY OF CALIFORNIA

MONTANA STATE UNIVERSITY

UNIVERSITY OF NEVADA, RENO

NEW MEXICO STATE UNIVERSITY

OREGON STATE UNIVERSITY

UNIVERSITY OF OREGON
UNIVERSITY OF SOUTHERN CALIFORNIA

STANFORD UNIVERSITY

UNIVERSITY OF HAWAII

UNIVERSITY OF TOKYO

UNIVERSITY OF UTAH

WASHINGTON STATE UNIVERSITY

UNIVERSITY OF WASHINGTON

The Supporting Institutions listed above contribute to the cost of publication of this Journal, but they are not owners or publishers and have no responsibility for its content or policies.

Mathematical papers intended for publication in the Pacific Journal of Mathematics should be in typed form or offset-reproduced, (not dittoed), double spaced with large margins. Please do not use built up fractions in the text of the manuscript. However, you may use them in the displayed equations. Underline Greek letters in red, German in green, and script in blue. The first paragraph or two must be capable of being used separately as a synopsis of the entire paper. Please propose a heading for the odd numbered pages of less than 35 characters. Manuscripts, in triplicate, may be sent to any one of the editors. Please classify according to the scheme of Math. Reviews, Index to Vol. 39. Supply name and address of author to whom proofs should be sent. All other communications should be addressed to the managing editor, or Elaine Barth, University of California, Los Angeles, California, 90024.

50 reprints to each author are provided free for each article, only if page charges have been substantially paid. Additional copies may be obtained at cost in multiples of 50 .

The Pacific Journal of Mathematics is issued monthly as of January 1966. Regular subscription rate: $\$ 84.00$ a year (6 Vols., 12 issues). Special rato: $\$ 42.00$ a year to individual members of supporting institutions.

Subscriptions, orders for numbers issued in the last three calendar years, and changes of address shoud be sent to Pacific Journal of Mathematics, P.O. Box 969, Carmel Valley, CA 93924, U.S.A Old back numbers obtainable from Kraus Periodicals Co., Route 100, Millwood, NY 10546.

PUBLISHED BY PACIFIC JOURNAL OF MATHEMATICS, A NON-PROFIT CORPORATION

Printed at Kokusai Bunken Insatsusha (International Academic Printing Co., Ltd.). 8-8, 3-chome, Takadanobaba, Shinjuku-ku, Tokyo 160, Japan.

Copyright (C) 1980 by Pacific Jounal of Mathematics Manufactured and first issued in Japan 


\section{Pacific Journal of Mathematics \\ Vol. 89, No. $2 \quad$ June, 1980}

Frank Hayne Beatrous, Jr. and R. Michael Range, On holomorphic

approximation in weakly pseudoconvex domains................. 249

Lawrence Victor Berman, Quadratic forms and power series fields ...... 257

John Bligh Conway and Wacław Szymański, Singly generated antisymmetric operator algebras ....................... 269

Patrick C. Endicott and J. Wolfgang Smith, A homology spectral sequence for submersions . . . .................................

Sushil Jajodia, Homotopy classification of lens spaces for one-relator groups with torsion ................................ 301

Herbert Meyer Kamowitz, Compact endomorphisms of Banach

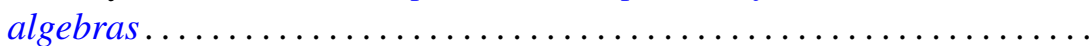

Keith Milo Kendig, Moiré phenomena in algebraic geometry: polynomial

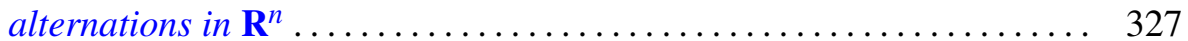

Cecelia Laurie, Invariant subspace lattices and compact operators....... 351

Ronald Leslie Lipsman, Restrictions of principal series to a real form . . . . . 367

Douglas C. McMahon and Louis Jack Nachman, An intrinsic

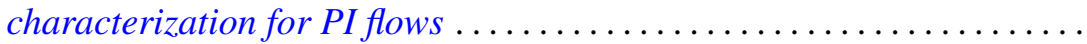

Norman R. Reilly, Modular sublattices of the lattice of varieties of inverse semigroups .................................... 405

Jeffrey Arthur Rosoff, Effective divisor classes and blowings-up of $\mathbf{P}^{2}$ 419

Zalman Rubinstein, Solution of the middle coefficient problem for certain

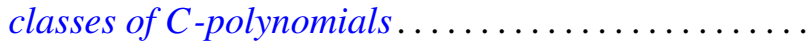

Alladi Sitaram, An analogue of the Wiener-Tauberian theorem for spherical transforms on semisimple Lie groups ................

Hal Leslie Smith, A note on disconjugacy for second order systems ...

J. Wolfgang Smith, Fiber homology and orientability of maps ...

Audrey Anne Terras, Integral formulas and integral tests for series of positive matrices. 